\title{
ADOLESCENTES NO ESPAÇO ESCOLAR E O CONHECIMENTO A RESPEITO DA SAÚDE SEXUAL E REPRODUTIVA*
}

\author{
Ediane de Andrade Ferreira1, Valdecyr Herdy Alves², Audrey Vidal Pereira ${ }^{3}$, \\ Diego Pereira Rodrigues ${ }^{4}$, Eny Dórea Paiva ${ }^{5}$, Inês Maria Meneses dos Santos ${ }^{6}$
}

\begin{abstract}
RESUMO: Objetivo: analisar o conhecimento de adolescentes sobre saúde sexual e reprodutiva no espaço escolar. Método: estudo descritivo, exploratório, de natureza qualitativa, realizado com 46 adolescentes de três escolas do ensino estadual do município de Macapá, estado do Amapá, no período de agosto a dezembro de 2016. A coleta de dados ocorreu por meio de entrevistas realizadas com a autorização dos respectivos responsáveis, e posteriormente foram transcritas e submetidas à análise de conteúdo na modalidade temática. Resultados: observou-se o conhecimento de adolescentes sobre a sexualidade relacionado ao ato sexual, à proteção contra gravidez, às doenças relacionadas ao sexo e à orientação sexual. Conclusão: as variadas expressões dos adolescentes permitiram evidenciar uma relação direta entre o ato sexual e os riscos de gravidez indesejada, o contágio por infecções sexualmente transmissíveis e a orientação sexual.

DESCRITORES: Adolescente; Sexualidade; Saúde Reprodutiva; Saúde Sexual; Enfermagem.

\section{ADOLESCENTS IN THE SCHOOL ENVIRONMENT AND KNOWLEDGE OF SEXUAL AND REPRODUCTIVE HEALTH *}

ABSTRACT: Objective: to analyze the knowledge of adolescents about sexual and reproductive health in the school environment. Method: qualitative exploratory descriptive study with 46 adolescents from three public schools of the state of Amapá, in the city of Macapá, from August to December 2016. Data was collected through interviews with the youngsters. The interviews were authorized by their parents or legal guardians and then transcribed and submitted to thematic content analysis. Results: Knowledge of the adolescents about sexuality associated with sexual intercourses, use of contraceptive methods, sex-related diseases and sexual orientation was analyzed. Conclusion: the various expressions of the adolescents revealed a direct relationship between sexual intercourse and the risks of unwanted pregnancy, and contamination by sexually transmitted infections and sexual orientation.

KEYWORDS: Adolescent; Sexuality; Reproductive Health; Sexual Health; Nursing.

\section{ADOLESCENTES EN EL AMBIENTE ESCOLAR Y EL CONOCIMIENTO ACERCA DE LA SALUD SEXUAL Y REPRODUCTIVA}

RESUMEN: Objetivo: analizar el conocimiento de adolescentes acerca de la salud sexual y reproductiva en el ambiente escolar. Método: estudio descriptivo, exploratorio, de naturaleza cualitativa, realizado con 46 adolescentes de tres escuelas de enseñanza estadual del municipio de Macapá, estado de Amapá, en el periodo de agosto a diciembre de 2016. Se recogieron los dados por medio de entrevistas realizadas con autorización de los respectivos responsables, siendo después transcriptas y sometidas al análisis de contenido en la modalidad temática. Resultados: se observó el conocimiento de adolescentes acerca de la sexualidad asociado al acto sexual, a evitar la gravidez, a las enfermedades relacionadas al sexo y a la orientación sexual. Conclusión: las distintas expresiones de los adolescentes posibilitaron evidenciar una relación directa entre el acto sexual y los riesgos del embarazo indeseado, el contagio por infecciones sexualmente transmisibles y la orientación sexual.

DESCRIPTORES: Adolescente; Sexualidad; Salud Reproductiva; Salud Sexual; Enfermería.

*Artigo extraído da dissertação intitulada: A expressão de adolescentes sobre sexualidade, saúde reprodutiva e métodos contraceptivos no município de Macapá, Universidade Federal Fluminense, 2017.

${ }^{1}$ Enfermeira. Mestre em Saúde Materno-Infantil. Professora Assistente da Universidade Federal do Amapá, AP - Brasil.

${ }^{2}$ Enfermeiro. Doutor em Enfermagem. Professor Titular da Escola de Enfermagem Aurora de Afonso Costa da Universidade Federal Fluminense. Niterói, RJ, Brasil.

${ }^{3}$ Enfermeiro. Doutor em Saúde Pública. Professor Adjunto da Escola de Enfermagem Aurora de Afonso Costa da Universidade Federal Fluminense. Niterói, RJ, Brasil.

${ }^{4}$ Enfermeiro. Mestre em Enfermagem. Professor Adjunto do Centro Universitário Anhanguera de Niterói do Curso de Enfermagem. Niterói, RJ, Brasil.

${ }^{5}$ Enfermeira. Doutora em Enfermagem. Professor Adjunta da Escola de Enfermagem Aurora de Afonso Costa da Universidade Federal Fluminense. Niterói, RJ, Brasil.

${ }^{6}$ Enfermeira. Doutora em Enfermagem. Professora Associada da Escola de Enfermagem Alfredo Pinto da Universidade Federal do Estado do Rio de Janeiro, Rio de Janeiro, RJ, Brasil. 


\section{INTRODUÇÃO}

A adolescência é definida como uma etapa da vida em que ocorrem inúmeras transformações biológicas, emocionais, psicológicas e sociais ${ }^{(1)}$. A Organização Mundial da Saúde (OMS) demarca essa fase da vida entre 10 e 19 anos de idade, enquanto o Estatuto da Criança e Adolescente (ECA) estabelece que esse período situa-se entre 12 e 18 anos incompletos ${ }^{(2)}$.

Para a OMS, a saúde sexual e reprodutiva dos adolescentes precisa de investigação científica e de Políticas Públicas específicas, pois $17,5 \%$ dos habitantes do mundo são adolescentes, e nos países periféricos esse grupo corresponde a $23 \%$ da população ${ }^{(3)}$. Diante dessa representatividade mundial, o Estado necessita de ações a fim de possibilitar uma mudança na saúde sexual e reprodutiva dos adolescentes.

A história da sexualidade relata que na Antiguidade, grega e romana, vivenciava-se uma liberdade sexual sem referência à noção de pecado ou moral, pois se vivia o completo prazer. O sexo era tanto para a reprodução como para a busca de sentimentos profundos de amor, para o prazer sexual e a sensualidade. Já na ascensão do Cristianismo, construiu-se uma moralidade permanente, na qual se mantinha a castidade ou o casamento, a recusa do prazer exclusivamente sexual, reduzindo assim as práticas sexuais aos limites estreitos dos interesses de procriação ${ }^{(4)}$.

$\mathrm{Na}$ atualidade, a sexualidade é um processo curiosamente atrativo, uma fase especial da vida, com inúmeras transformações hormonais que se exacerbam nos adolescentes pelas descobertas da própria sexualidade. Todavia, a falta ou o pouco conhecimento desse grupo populacional a respeito da temática estão relacionados com o aumento de gravidez na adolescência e de Infecções Sexualmente Transmissíveis (IST) $)^{(5)}$.

Mediante tal contexto, torna-se necessário trilhar um caminho que apoie efetivamente os adolescentes em suas necessidades, que Ihes permita um acesso rápido à informação, empoderando-os por meio de informações corretas para que haja a participação de cada um na vida sexual e reprodutiva de forma segura e satisfatória, possibilitando, assim, a liberdade de decisão, livre de discriminação, coerção e violência $^{(6)}$. Além disso, é imprescindível que se garanta a elaboração e a aplicabilidade de Políticas Públicas, e que se crie serviços de saúde cujas medidas do Estado em prol da saúde sexual e reprodutiva do adolescente estejam ancoradas nos direitos deste.

Nesse sentido, pontua-se a necessidade de executar o Plano Curricular Nacional no que tange à sexualidade no ambiente escolar, já que é uma temática presente no cotidiano de estudantes, pais e professores. Assim, poderão ser debatidos temas importantes para a saúde dos adolescentes, em especial a garantia da autonomia de suas escolhas e a prevenção de IST e de gravidez indesejada a partir do processo informativo da educação ${ }^{(7)}$.

Para tanto, são necessárias estratégias relacionadas à informação dos adolescentes, em especial direcionadas àqueles em período escolar, com vistas à promoção de uma discussão crítica e reflexiva, extensiva aos seus responsáveis e professores. Entretanto, existem entraves relacionados ao direito dos adolescentes de vivenciar a sexualidade com menos conflitos e intercorrências. Podem ser observados dilemas no sentido de conciliar processo de independência e capacidade de tomar decisões, portanto, há a necessidade de proteção desses indivíduos, que, por exemplo, encontram-se potencialmente vulneráveis às $\operatorname{IST}^{(8)}$.

No contexto geográfico desta pesquisa, realizada no município de Macapá, capital do Estado do Amapá, o governo estadual deve promover medidas que abordem os direitos sexuais e reprodutivos, com informação, acompanhamento, oferta de métodos contraceptivos e serviços de saúde. Também deve ser fornecida capacitação de profissionais de saúde e professores para que estes possam lidar com os adolescentes, quebrando o tabu de falar sobre sexualidade e mostrando a importância do conhecimento e exercício dos direitos sexuais e reprodutivos.

Os índices emitidos pelo Serviço de Arquivo Médico e Estatística do Hospital da Mulher Mãe Luzia, referência em gestação de alto risco no estado de Macapá, mostram que, no período de 2011 a 2015, houve uma taxa crescente nos atendimentos específicos de mulheres de 10 a 19 anos, e que 9.322 partos ocorreram por frequência absoluta. Ainda, no mesmo período, 1.306 adolescentes foram submetidas 
a curetagens uterinas. A soma por frequência relativa do número de gestações e curetagens realizadas anualmente no período apresentado mostra uma variação de 38,21\% (2011) a 48,47\% (2015), evidenciando que quase $50 \%$ dos atendimentos da maternidade foram de adolescentes. Dessa forma, torna-se necessário intervir nessa problemática, em especial com ações nos serviços de atenção básica, e principalmente no ambiente escolar, a partir da abordagem da dimensão da saúde sexual de forma segura ${ }^{(5)}$.

Nessa perspectiva, este estudo teve como objetivo analisar o conhecimento de adolescentes sobre saúde sexual e reprodutiva no espaço escolar.

\section{- MÉTODO}

Estudo descritivo, exploratório, de natureza qualitativa, realizado em três escolas estaduais de ensino médio, situadas no município de Macapá, estado do Amapá, Brasil, no período de agosto a dezembro de 2016.

A escolha dos locais ocorreu por meio de processo aleatório simples, obtendo-se uma escola por região, a saber: Escola Estadual Professora Sebastiana Lenir de Almeida (Região Sul), Escola Estadual Professor Alexandre Vaz Tavares (Região Central) e Escola Estadual Professora Maria Ivone de Menezes (Região Norte).

Os participantes do estudo foram 46 estudantes que atenderam aos seguintes critérios de inclusão: estar na faixa etária de 13 a 18 anos de idade e estar matriculado regularmente na rede estadual de ensino. O critério de exclusão correspondeu a adolescentes com algum problema de ordem física, emocional ou psicológica que comprometesse a sua participação. Foi realizada entrevista semiestruturada sobre a saúde sexual e reprodutiva, que ocorreu em ambiente privado, assim garantindo a privacidade do participante. As entrevistas cessaram por meio do processo de saturação dos dados.

Os depoimentos foram coletados por aparelho digital, transcritos na íntegra e submetidos à análise de conteúdo na modalidade temática ${ }^{(9)}$. Para assegurar o sigilo e o anonimato do depoimento dos participantes, estes foram identificados como 'Adolescentes' e receberam um código alfanumérico sequencial (A1, A2,... A46). Foi utilizada a unidade de registro a partir da temática como estratégia de organização do conteúdo das entrevistas. Para tanto, selecionaram-se cores distintas, com a finalidade de identificar cada unidade e agrupá-las, possibilitando, assim, uma visão geral da temática. As seguintes unidades de registro foram originadas: Sexualidade como ato sexual; Ato que protege contra a gravidez; Coisa de homem e mulher; Evitar doenças do sexo; Orientação sexual. Essas unidades de registro fundamentaram a construção da seguinte categoria temática: O conhecimento de adolescentes sobre a sexualidade - um desafio para a escola.

A pesquisa foi aprovada pelo Comitê de Ética em Pesquisa do Hospital Universitário Antônio Pedro, da Universidade Federal Fluminense, sob protocolo n.ำ 1.349.794/2015, conforme dispõe a Resolução n. ${ }^{\circ}$ 466/2012 do Conselho Nacional de Saúde ${ }^{(10)}$. Para concretizar a participação, todos os adolescentes assinaram o Termo de Assentimento Informado, complementado com a autorização dos respectivos responsáveis e/ou representantes legais, os quais assinaram o Termo de Consentimento Livre Esclarecido.

\section{RESULTADOS}

\section{O conhecimento de adolescentes sobre a sexualidade - um desafio para a escola}

Nesta categoria, o conhecimento sobre sexualidade dos adolescentes foi relacionado com: ato sexual, proteção contra gravidez, coisa de homem e mulher, evitar doenças do sexo e orientação sexual, conforme relatos a seguir:

Tenho dúvidas sobre isso. Seriam relações sexuais? (A13)

Serve para fazer filho, para ter prazer, complementar as relações amorosas. (A22) 
Sexualidade é falar sobre sexo, só ouvi falar, só isso mesmo. (A27)

É um ato bem íntimo, e não acho, portanto, uma coisa de risco, depende da pessoa. (A30)

A correlação da sexualidade com a homossexualidade ficou entre as percepções mais observadas:

É o ato de ter relações sexuais com o sexo oposto. E também tem pessoas que tem relação com pessoas do próprio sexo. (A7)

Tipo sexualidade, no caso heterossexual como pessoas tenham bi, gays, lésbicas. E diversificando as sexualidades. (A17)

A sexualidade, já ouvi falar sobre sexo, homossexuais, só isso. (A20)

Sexualidade em si ou opção sexual, por sexualidade eu não sei especificar bem, mas acho que é opção sexual, gostar de homem ou mulher. (A25)

O modelo compreendido relaciona a sexualidade ao processo saúde e doença e à prevenção de agravos, o que é evidenciado nos depoimentos dos estudantes quando associam apenas os componentes biológicos e a prevenção da gravidez indesejada, conforme os padrões sociais vigentes:

Isso, pra mim tem que se prevenir muito, porque muita gente não tem dinheiro, aí fica assim, não sabe o que é pegar coisas nas costas. (A23)

Tem que usar preservativo para não engravidar, e uns quando querem engravidar não usam preservativos, e uns, mesmo sabendo, não usam. (A32)

Entendo bem pouca coisa, eu entendo que sempre tem que se prevenir para não engravidar e para não ser abalado por doenças também, que tem na sexualidade. (A37)

Bem, meio que no ano passado, eu estudei sobre isso, mas não lembro direito. Pelo que entendi, o professor de Ciências passou para a gente sobre os meios de não engravidar. (A40)

Enquanto alguns estudantes observam a sexualidade como orientação sexual, relações heterossexuais, bissexualidade e homossexualidade, outros percebem como ato sexual exclusivamente ancorado na visão binária homens/mulheres:

Eu entendo que é uma relação entre homens e mulheres. Por exemplo, eu estou namorando, aí a gente fica à vontade assim no quarto e, se der vontade, a gente vai se relacionando mais e fazendo outras coisas. (A12)

É a relação entre o homem e uma mulher. (A18)

Sexualidade é... não muita coisa, visão de gênero masculino e feminino e, em geral, eu. Cara! é muita coisa, e eu não tenho como especificar, sabe? (A26)

É a relação que tem entre um homem e uma mulher para gerar uma vida. (A33)

Novamente, os componentes biológicos refletem-se nos depoimentos dos estudantes, e a relação entre sexualidade e prevenção de IST leva à inferência de que talvez o processo de formação desses estudantes esteja sendo conduzido apenas pela ideia de riscos. Neste caso, as infecções são causadas pelo sexo desprotegido:

Ouvi falar muitas coisas, assim, mas eu não tenho muito conhecimento a dizer. Eu tenho umas coisas na cabeça, mas nada muito específico. Doenças sexualmente transmitidas. (A17)

Eu entendo que a sexualidade não é só fazer amor. Acho que é entre homem e mulher. Que tem uma área que pode ou não, tem outras formas de se cuidarem, pois podem pegar doenças contagiosas. Eu acho que sexualidade é isso. (A24)

Seria o que a gente tem a tratar durante a sexualidade, não fazer sexo com qualquer parceira, ver se ela não tem nenhuma doença transmissível, usar camisinha, fazer prevenção. (A37) 
Percebe-se que sexualidade e saúde reprodutiva, de acordo com os depoimentos a seguir, são abordadas praticamente nas disciplinas de Ciências e Biologia e em palestras realizadas nas escolas pelas equipes de Saúde da Família:

Na escola, só na aula de Ciência sobre DST. (A12)

Bom, os profissionais, como os professores de Biologia, sempre estão dispostos a responder essas coisas de relação sexual. (A29)

Na escola e com meus pais. Tenho aula de Ciências. (A30)

Bem, no meio do ano passado eu estudei sobre isso, mas não lembro direito. Pelo que entendi, o professor de Ciências passou para a gente sobre os meios pra não engravidar, sobre como ter relações sexuais, meio que lá na frente a pessoa ter um problema com gravidez ou outras coisas. (A31)

\section{DISCUSSÃO}

\section{A sexualidade diz respeito a um:}

conjunto de características humanas, que se traduz nas diferentes formas de expressar a energia vital, chamada por Freud de 'libido', que quer dizer energia pela qual se manifesta a capacidade de se ligar às pessoas, ao prazer/desprazer, aos desejos, às necessidades, à vida. Ao refletir um pouco mais sobre o assunto, pode-se perceber que o corpo como um todo é fonte de prazer, pelo fato de propiciar, desde o nosso nascimento, o sentir, o perceber e o comunicar o mundo ${ }^{(11: 39)}$.

A vulnerabilidade dos jovens no campo da sexualidade é mostrada por alguns indicadores, tais como desconhecimento sobre ciclos reprodutivos, gravidez juvenil, preservativos, conversa com adultos, limites individuais e discriminações por conta de gênero e de orientação sexual ${ }^{(12)}$. Esses indicadores correspondem às dificuldades que os adolescentes têm de compreender de forma satisfatória a própria saúde sexual.

A sexualidade, especialmente na adolescência, é um componente intrínseco da pessoa, é fundamental na saúde dos jovens porque transcende o aspecto meramente biológico, manifestando-se também como um fenômeno psicológico e social, fortemente influenciado por crenças e valores pessoais e familiares, normas morais e tabus sociais ${ }^{(13)}$. Desse modo, a sexualidade deve ser discutida como um todo, envolvendo adolescentes, responsáveis, professores, profissionais de saúde e sociedade, para favorecer o diálogo e o conhecimento, assim contribuindo para minimizar os fatores de vulnerabilidade.

A questão da visibilidade da homossexualidade na educação brasileira torna-se urgente. Uma pesquisa da Organização das Nações Unidas para a Educação, a Ciência e a Cultura (UNESCO) sobre juventude e sexualidade, envolvendo estudantes brasileiros do ensino fundamental, seus responsáveis e professores, revelou que todos eles não apenas silenciam a discriminação de homossexuais, como também colaboram ativamente para a reprodução dessa violência ${ }^{(12)}$.

Dentro do espaço escolar, as medidas educacionais sobre a homossexualidade são apáticas, assim, a tendência é considerá-la uma anomalia. A homossexualidade não é discutida e está centrada em estereótipos, e, dessa forma, a ordem hierárquica é mantida por meio de um discurso que reforça a intolerância. O desejo é fragmentado, o debate centra-se em torno das causas da orientação sexual, em um duelo opositor entre natureza e cultura, sem considerar a diversidade de aspectos que envolvem a sexualidade humana ${ }^{(14)}$.

Assim, mais que ressaltar níveis estatísticos, vale o alerta de que essas vulnerabilidades existem, e "ferem", "doem", "magoam" e "comprometem" o que pode ser um dos construtos mais ricos e importantes da vida dos seres humanos, no que se refere ao processo de construção identitária dos jovens: a sexualidade, principalmente se entendida como elo de vida, afetividade e sentimentos, algo para além da genitalidade ${ }^{(12)}$. 
A sexualidade precisa ser compreendida no âmbito da história e da cultura. Nessa ótica, as identidades sexuais deixam de ser concebidas como meros resultantes de "imperativos biológicos"(14), passando a ser entendidas como constituídas nas relações sociais de poder, em complexas articulações e em múltiplas instâncias sociais ${ }^{(15)}$.

A educação sexual, relacionada à sexualidade, oferecida pela escola, deve abordar, juntamente com as crianças e os jovens, as repercussões de todas as mensagens transmitidas pela mídia, pela família e pela sociedade. Trata-se de preencher lacunas nas informações que os estudantes já possuem, e, principalmente, de criar a possibilidade de formar opinião a respeito do que thes é ou foi apresentado ${ }^{(15)}$.

Trabalhar Orientação Sexual significa ampliar e aprofundar a visão sobre a sexualidade, ressaltando os diferentes pontos de vista existentes na sociedade, incluindo as práticas sexuais ligadas ao afeto, ao prazer, ao respeito e à própria sexualidade. Tal trabalho não deve se limitar exclusivamente à mera informação reprodutiva ou preventiva, mas abranger uma dimensão histórica, cultural, ética e política que envolve todo o ser: corpo e espírito, razão e emoção, ou seja, a sexualidade pode ser expressa de diversas maneiras: carícias, beijos, abraços e olhares. Portanto, a orientação sexual deve compreender o desenvolvimento sexual como saúde reprodutiva, relações de gênero, relações interpessoais, afetivas, imagem corporal e autoestima ${ }^{(16)}$.

Problematizar as construções sociais do masculino e do feminino em uma proposta pedagógica é uma forma de contribuir para a equidade de gênero, pois as diferenças biológicas servem de argumento para justificar uma série de desigualdades sociais entre homens e mulheres. Por sua vez, tais desigualdades formam a base para justificar o comportamento de ambos ${ }^{(17)}$.

Nesse sentido, a instituição escolar, pela ação de seus atores, desempenha papel especial na produção de identidades sexuais e de gênero, assim como na validação de determinadas formas de viver as masculinidades, as feminilidades e as sexualidades ${ }^{(18)}$.

Reconhecer a sexualidade como construção social significa compreender que as práticas e os desejos são também construídos culturalmente, dependendo da diversidade de povos, concepções de mundo e costumes existentes, mesmo quando essa diversidade é abrangida por um só país, como ocorre no Brasil $^{(19)}$.

Nas escolas, percebe-se que se instalaram certos dispositivos para regular e gerir a sexualidade dos estudantes. Assim, essa temática é discutida a fim de evitar uma gravidez na adolescência e prevenir o contágio de IST, principalmente da Acquired Immunodeficiency Syndrome (AIDS). Desse modo, as instituições educacionais vêm normatizando os corpos dos indivíduos e o corpo social(7).

A mobilização em torno da administração da sexualidade adolescente desemboca em diversas formas de institucionalização do problema. A rede escolar passa a ser pensada como um dispositivo político de intervenção, privilegiando e buscando expandir o impacto sobre a população por meio do controle da sexualidade de crianças, principalmente de adolescentes. No entanto, esse processo deve transcender o acesso às informações sobre controle de natalidade e práticas preventivas, deve formar sujeitos críticos e reflexivos, que vivam a iniciação de sua vida sexual com autorresponsabilidade, prevenindo-se da gravidez e dos perigos trazidos pelas IST ${ }^{(7)}$.

Assim, os depoimentos dos adolescentes expressaram conceitos que permitiram observar que temas como sexualidade e saúde reprodutiva não estão sendo conduzidos com transversalidade no âmbito da educação, como é proposto no Plano Curricular Nacional.

A transversalidade pressupõe um tratamento integrado das áreas e um compromisso das relações interpessoais e sociais escolares com as questões que estão envolvidas nos temas, a fim de que haja coerência entre os valores experimentados na vivência que a escola propicia aos estudantes e o contato intelectual com tais valores ${ }^{(20)}$.

Nesse sentido, os Parâmetros Curriculares Nacionais trazem a proposta da sexualidade e outras questões sociais e/ou atuais que devem compor as discussões realizadas na escola, pois tais questões estão presentes no cotidiano dos professores e estudantes, portanto, são vivenciadas por eles. Além disso, a proposta desses Parâmetros é a discussão da orientação sexual na escola não apenas em uma disciplina específica, instituindo uma voz autorizada, mas como um tema que perpasse todas as áreas do saber. Assim, temáticas acerca de corpos, gêneros e sexualidades estão inseridas nas questões sociais, 
fazem parte do cotidiano das escolas, seja na separação das filas entre meninas e meninos, seja nos namoros no corredor ou na gravidez de uma adolescente ${ }^{(7)}$.

As Diretrizes e Bases da Educação Brasileira Nacional (LDBEN), por meio da Lei n.․ 9.394/1996, regulamentaram o direito à educação como um direito público subjetivo de todo cidadão e estabeleceram que compete à União Federal elaborar o Plano Nacional de Educação, em colaboração com Estados, Distrito Federal e Municípios, outorgando ao Conselho Nacional de Educação as funções normativas e de supervisão. Assim, e considerando a descentralização política e administrativa definida na Constituição de 1988, os Parâmetros Curriculares Nacionais apresentam referenciais para a elaboração descentralizada de propostas curriculares em níveis estadual e municipal, incluindo a saúde e a sexualidade entre os temas a serem abordados de forma transversal no currículo escolar ${ }^{(21)}$.

\section{CONCLUSÃO}

Os estudos sobre adolescentes e suas condições sociais, demográficas e biológicas, em específico sobre sexualidade, saúde reprodutiva e conhecimento dos métodos contraceptivos, são comuns na realidade brasileira. Porém, considerando os tabus que ainda cercam as temáticas, torna-se necessário enfrentar várias dificuldades na execução de ações e pesquisas qualitativas nas instituições escolares.

O estudo mostrou os variados conhecimentos dos adolescentes a respeito da sexualidade. Verificouse, por meio dos depoimentos, que existe uma relação direta entre o ato sexual e o risco da gravidez indesejada, o contágio por doenças sexualmente transmissíveis entre homens e mulheres ou relações homossexuais e a ideia de orientação sexual.

Além disso, a abordagem sobre saúde reprodutiva não foi identificada como tema regular, oferecido com linguagem clara e multidisciplinar, mas ela ainda é repassada de forma tradicional, considerando as expressões e a realidade dos estudantes. Pode-se afirmar que a ausência de uma abordagem transversal e intersetorial expressam respostas vagas ou mesmo equivocadas dos estudantes com relação à própria sexualidade. Desse modo, para abordar a saúde sexual e reprodutiva, torna-se necessário falar dos direitos sexuais e dos direitos reprodutivos, de modo que sejam trabalhadas questões de modo crítico e reflexivo no processo de educação e saúde.

\section{- REFERÊNCIAS}

1. Dantas LA, de Andrade LDF, de Lima GMB, Saraiva AM. O desabrochar das flores: opiniões de adolescentes grávidas sobre planejamento familiar. Cogitare Enferm. [Internet] 2013;18(3) [acesso em 01 out 2017]. Disponível: http://dx.doi. org/10.5380/ce.v18i3.33563.

2. Senna SRCM, Dessen MA. Reflexões sobre a saúde do adolescente brasileiro. Psicol. saúde doenças [Internet] 2015;16(2) [acesso em 01 Out 2017]. Disponível: http://dx.doi.org/10.15309/15psd160208.

3. Ministério da Saúde (BR). Secretaria de Atenção à Saúde. Departamento de Ações Programáticas Estratégicas. Política Nacional de Atenção Integral à Saúde da Mulher: princípios e diretrizes. Brasília: Ministério da Saúde; 2011.

4. Marola CAG, Sanches CSM, Cardoso LM. Formação de conceitos em sexualidade na adolescência e suas influências. Psicol. educ. [Internet] 2011;(33) [acesso em 01 out 2017]. Disponível: http://pepsic.bvsalud.org/scielo.php?script=sci_ arttext\&pid=S1414-69752011000200006.

5. Ferreira EA. A expressão de adolescentes sobre sexualidade, saúde reprodutiva e métodos contraceptivos no município de Macapá [dissertação]. Niterói (RJ): Universidade Federal Fluminense; 2017.

6. Ministério da Saúde (BR). Secretaria de Vigilância em Saúde. Adolescentes e jovens para a Educação entre pares: saúde e prevenção nas escolas. Brasília: Ministério da Saúde; 2010. 
7. de Barros SC, Ribeiro PRC. Educação para a sexualidade: uma questão transversal ou disciplinar no currículo escolar? Revista Electrónica de Enseñanza de las Ciencias. [Internet] 2012;11(1): [acesso em 01 out 2017]. Disponível: http:// reec.uvigo.es/volumenes/volumen11/REEC_11_1_9_ex570.pdf.

8. Taquette SR. Direitos sexuais e reprodutivos na adolescência. Adolesc. Saúde [Internet] 2013;10(Suppl 1) [acesso em 01 Out 2017]. Disponível: http://www.adolescenciaesaude.com/detalhe_artigo.asp?id=397.

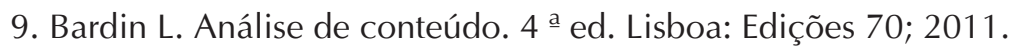

10. Ministério da Saúde (BR). Conselho Nacional de Saúde. Diretrizes e normas regulamentadoras de pesquisa envolvendo seres humanos. Resolução n. 466, de 12 de dezembro de 2012. Brasília; 2012.

11. Ministério da Saúde (BR). Secretaria de Atenção à Saúde. Departamento de Atenção Básica Saúde sexual e saúde reprodutiva. Brasília DF: Ministério da Saúde; 2013.

12. de Moraes SP, Vitalle MSS. Direitos sexuais e reprodutivos na adolescência: interações ONU-Brasil. Cienc. saúde colet. [Internet] 2015;20(8) [acesso em 01 Out 2017]. Disponível: http://dx.doi.org/10.1590/1413-81232015208.03112014.

13. Ministério da Saúde (BR). Secretaria de Atenção em Saúde. Departamento de Ações Programáticas Estratégicas. Diretrizes nacionais para a atenção integral à saúde de adolescentes e jovens na promoção, proteção e recuperação da saúde. Brasília: Ministério da Saúde; 2010.

14. Britzman DP.Professor@s e Eros. Educ. rev. [Internet] 2009;(35) [acesso em 01 out 2017]. Disponível: http://dx.doi. org/10.1590/S0104-40602009000300005.

15. Ministério da Saúde (BR). Secretaria de Vigilância em Saúde. Saúde e prevenção nas escolas: guia para formação de profissionais da saúde e de educação. Brasília: Ministério da Saúde; 2007.

16. Gonçalves RC, Faleiro JH, Malafaia G. Educação sexual no contexto familiar e escolar: impasses e desafios. HOLOS. [Internet] 2013;(5) [acesso em 01 Out 2017]. Disponível: https://doi.org/10.15628/holos.2013.784.

17. Altmann H, Martins CJ. Educação sexual: ética, liberdade e autonomia. Educ. Rev. [Internet] 2009;(35) [acesso em 01 out 2017]. Disponível: http://dx.doi.org/10.1590/S0104-40602009000300006.

18. Quirino GS, da Rocha JBT. Sexualidade e educação sexual na percepção docente. Educ. Rev. [Internet] 2012;(43) [acesso em 01 out 2017]. Disponível: http://dx.doi.org/10.1590/S0104-40602012000100014.

19. Barros JPP, Calaço VFR. "Meu prazer agora é risco": sentidos sobre sexualidade entre jovens de um grupo sobre saúde. Fractal, Rev. Psicol. [Internet] 2013;25(1) [acesso em 01 out 2017]. Disponível: http://dx.doi.org/10.1590/S198402922013000100005.

20. Barbosa MR. A aprendizagem histórica e os professores na história. Curitiba: Appris; 2017.

21. Ministério da Saúde (BR). Guia de sugestões de atividades Semana Saúde na escola. Tema: Sexualidade e saúde reprodutiva. Brasília: Ministério da Saúde; 2013. 